\section{Editor-in-Chief \\ Barbara McLain - (retired Prof.) \\ University of Hawaii, USA}

The Israeli Journal of Aquaculture (IJA) is an interdisciplinary journal that is dedicated to sharing new research and tested applications of aquaculture

The IJA is devoted to scholarly articles for improved aquaculture practices and related industries

The IJA is a peer-reviewed, open-access, electronic journal

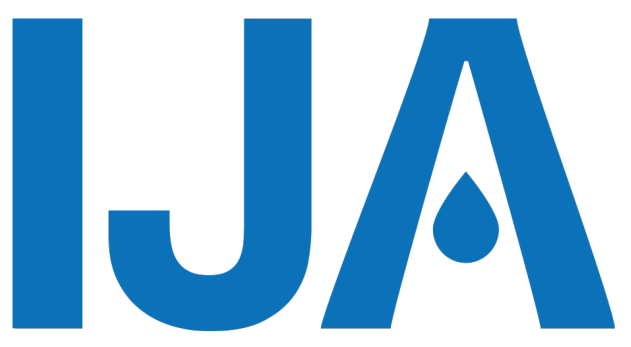

\section{The () Israeli Journal of Aquaculture}

An interdisciplinary online Open Access scientific journal

Published by the

\section{AquacultureHub}

A non-profit organization 501c3

http://www.aquaculturehub.org

in partnership with the

\section{University of Hawaii at Manoa} Library

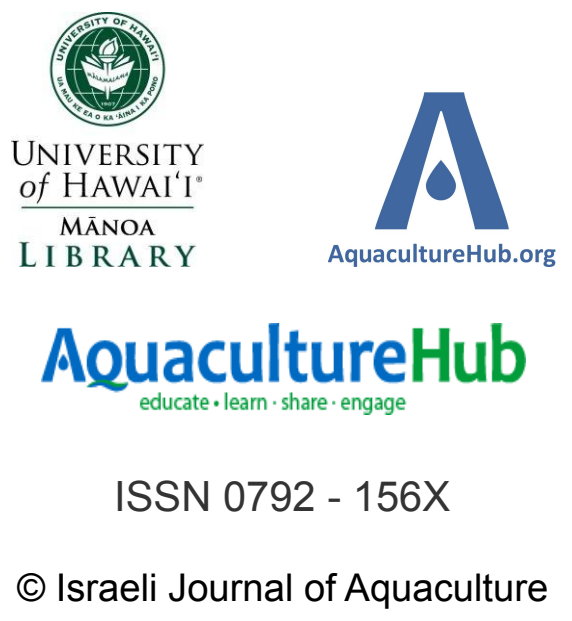




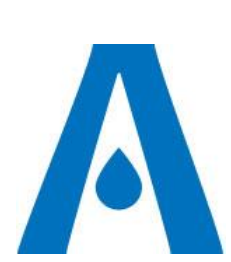

Produced by the AquacultureHub non-profit Foundation the $I J A$ is an open-access, scientific journal, published on http://www.aquaculturehub.org/group/israelijournalofaq uaculturebamidgehija

To read papers free of charge, please register online at the above website.

Sale of IJA papers is strictly forbidden.

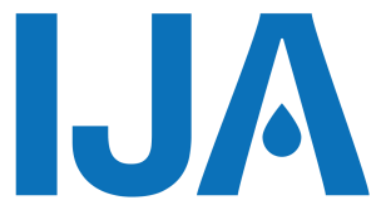

\title{
Nitrite-Induced Oxidative Stress, Histopathology, and Transcriptome Changes in the Mud Crab (Scylla paramamosain)
}

\author{
Chang-Hong Cheng ${ }^{1}$, You-Lu Su${ }^{1}$, Hong-Ling Ma ${ }^{1}$, Yi-Qin Deng ${ }^{1}$, \\ Juan Feng ${ }^{1}$, Xiao-Long Chen ${ }^{1}$, Zhi-Xun Guo ${ }^{1,2 *}$ \\ ${ }^{1}$ Key Laboratory of South China Sea Fishery Resources Exploitation \& \\ Utilization, Ministry of Agriculture, South China Sea Fisheries \\ 2 Research Institute, Chinese Academy of Fishery Sciences, Guangzhou, \\ Guangdong 510300, China, PR China
}

Keywords: Scylla paramamosain; nitrite stress; oxidative stress; transcriptomic analysis

\begin{abstract}
Nitrite in the aquatic environment is highly toxic to aquatic animals. However, the mechanism by which the mud crab responds to nitrite-induced stress remains unclear. In this study, we investigated the physiological response and molecular mechanism in the mud crab (Scylla paramamosain) exposed to the acute nitrite exposure ( $20 \mathrm{mg} / \mathrm{L}$ ) for $24 \mathrm{~h}$. The results showed that nitrite exposure induced significant changes in antioxidant enzyme activity and MDA content. Severe cytological damage was observed in the hepatopancreas. After $24 \mathrm{~h}$ exposure to nitrite, 11,638 differentially expressed genes (DEGs) were identified by transcriptome analysis. These DEGs were involved in many pathways related to oxidative stress and immune responses. Our results also found that FoxO signaling pathway, p53 signaling pathway, and NF-kB signaling pathway participated in the anti-stress defense against nitrite stress. The study provides new insight into the understanding of nitrite-induced toxicity in the mud crab.
\end{abstract}

\footnotetext{
* Corresponding author. Z-X Guo, Tel./fax: +86 2089108331; email address: guozhixun1@163.com
} 


\section{Introduction}

Nitrite is an intermediate product of bacterial denitrification of nitrate or bacterial nitrification of ammonia (Hooper et al., 1997). Nitrite concentration is very low in seawater. However, nitrite concentration can build up in intensive aquaculture systems. Elevated nitrite in aquaculture systems can result in accumulated uptake of nitrite across gill epithelium and cause very high concentrations in the body fluids (Jensen et al., 2003). Thus, the high level of nitrite in water is a potential factor triggering stress response in aquatic organisms (Palachek and Tomasso, 1984; Jia et al., 2015; Chand and Sahoo, 2006).

Previous studies showed that nitrite exposure adversely affects growth (Chen and Chen, 1992), endocrine process (Deane and Woo, 2007), osmoregulatory function (Woo and Chiu, 1997), and ammonia excretion (Chen and Chen, 1995a). In crustaceans, elevated nitrite in aquaculture systems have been reported to reduce the level of hemocyanin (Chen and Chen, 1995), depress immunologic function (Wang et al., 2004), and increase vulnerability to disease (Tseng and Chen, 2004). Many studies have reported that oxidative stress is one of the mechanisms of toxicity of nitrite on aquatic organisms (Xian et al., 2011; Romano and Zeng, 2013; Guo et al., 2013). When the organism is incapable of dealing with production of reactive oxygen species (ROS), oxidative stress occurs (Lesser, 2006). Oxidative stress can trigger DNA damage, protein oxidation, lipid peroxidation, and a decline of physiological function (Stadtman and Levine, 2003). To prevent the damage caused by environmental stress, organisms have developed several defensive responses such as antioxidant defense. Low levels of nitrite have been shown to increase the expression of antioxidant enzymes in red claw crayfish (Jiang et al. 2014).

Transcriptome profiling analysis is a rapid and effective approach for genome studies and functional gene identification. Ribonucleic acid sequencing (RNA-Seq) is a relatively new technology for transcriptomic studies which provides information on physiological signalling pathways and the gene regulation networks underlying biological processes (Mortazavi et al., 2008). Thus, RNA-Seq has been widely utilized to investigate transcriptome responses of aquatic animals to ambient stress (Guo et al., 2013; Wang et al., 2016). The RNA-seq analysis of the fish Megalobrama amblycephala exposed to nitrite stress has identified numerous candidate genes associated with oxidative stress, oxygen transport, immune responses, and the metabolism of proteins and fats (Sun et al., 2014). Apoptosis and immune related pathways under nitrite stress were identified in oriental river prawn by RNA-Seq (Yu et al. 2019). However, limited studies have reported the molecular mechanism of the mud crab exposed to high environmental nitrite.

The mud crab (Scylla paramamosain) is widely distributed along the coast in South China. Mud crab cultivation has become increasingly popular in South China. However, the expansion of mud crab aquaculture has brought been accompanied by great challenges including devastating diseases resulting in large economic losses (Weng et al., 2007; Guo et al., 2013). Environmental pollutants also increase vulnerability to disease. Thus, in this study, we focused on the effects of nitrite exposure on gene expression, hematological parameters, and histological alterations in the mud crab. These results will provide new insights into understanding nitrite-induced toxicity in the mud crab.

\section{Materials and methods}

Mud crabs (weight $100 \pm 6 \mathrm{~g}$ ) were obtained from aquaculture facilities in the providence of Guangdong providence. They were approximately four months old, maintained in tanks at $10 \%$ salinity and fed a diet of oyster meat twice daily. The crabs were fasted for $24 \mathrm{~h}$ prior to experimental treatments. During the experimental period, water temperature was $25^{\circ} \mathrm{C}, \mathrm{pH}$ was 7.8 , dissolved oxygen was not less than $6.0 \mathrm{mg} / \mathrm{L}$, and ammonia nitrogen was lower than $0.05 \mathrm{mg} / \mathrm{L}$.

Our preliminary studies demonstrated that after 96-hour LC50 nitrite was approximately $40 \mathrm{mg} / \mathrm{L}$ (unpublished). We conducted a preliminary experiment to determine the optimal concentration of nitrite and sampling time point for physiology and transcriptome analysis. Based on these results, mud crabs exposed to $1 / 2$ of the $96 \mathrm{~h}$ LC50 (20 mg/L) for $24 \mathrm{~h}$ were considered appropriate samples for subsequent physiology and transcriptome analysis. Mud crabs were randomly divided into six $200 \mathrm{~L}$ tanks with 
30 individuals per unit. The nitrite concentration of each exposure group ( 0 and $20 \mathrm{mg} / \mathrm{L}$ ) was adjusted to the desired concentration by adding $\mathrm{NaNO}_{2}$ to $10 \%$ saltwater. There were three replicates per group. During the exposure experiment, the nitrite concentration was tested using the Griess method (Federation and Association, 2005), and adjusted by adding $\mathrm{NaNO}_{2}$ solution every $12 \mathrm{~h}$. After $0,6,12$ and $24 \mathrm{~h}$ exposure, nine mud crabs were randomly sampled from each group. Hemolymph of individual mud crabs was withdrawn from the base of the third pereiopod using a $1 \mathrm{ml}$ sterile syringe (25 gauge). The hemolymph was centrifuged at $8000 \times \mathrm{g}$ at $4^{\circ} \mathrm{C}$ for $30 \mathrm{~min}$. The supernatant fluid was collected to analyze superoxide dismutase (SOD) activity, catalase (CAT) activity, and malondialdehyde (MDA). After collecting hemolymph, hepatopancreas samples were removed and stored at $-80^{\circ} \mathrm{C}$ for later analysis.

The activities of SOD, CAT and MDA assays were measured using commercially available kits (Nanjing Jiancheng Chemical Industries, Nanjing, Jiangsu, China) following the instructions of the manufacturer. CAT activity was determined according to the method of Aebi (1984), which involved $\mathrm{H}_{2} \mathrm{O}_{2}$ breakdown. SOD activity was measured according to the method of Peskin and Winterbourn (2000), based on the oxidation of epinephrine adrenochrome transition by the enzymes. Lipid peroxidation was analyzed by a thiobarbituric acid reactive substances assay (Ohkawa et al., 1979).

Hepatopancreas samples from six mud crabs of each group were collected for histopathologic examination. Histopathological changes were examined under a light microscope. Histological alterations (10 random microscope fields/section, 1 sections/sampling, and 6 samplings/each group) were evaluated using degree of tissue change based on the lesion severity. Tissues were fixed in $10 \%$ formaldehyde, cleared in xylene, and embedded in paraffin. Sections $(5 \mu \mathrm{m})$ were cut, then stained with hematoxylin and eosin. Stained samples were observed under a light microscope.

Total RNA was isolated from hepatopancreas using TRIzol reagent (Invitrogen, USA) according to the manufacturer's instructions. The quantity and integrity were assessed by an Agilent 2100 bioanalyzer. The RIN (RNA integrity number) value was 6.07.5. RNA from three individuals (one per biological replicate) of each group was pooled for constructing a sequencing library. The mRNA was purified using TruSeq RNA Sample Prep Kit (Illumina, USA) following the manufacture's instruction, and then fragmented. The CDNA libraries were prepared with SuperScript II reverse transcriptase kit (Invitrogen, USA) and amplified with PCR using a TruSeq PE Cluster kit (Illumina, USA). The final quality of the fragments was checked by an Agilent 2100 Bioanalyzer. The cDNA libraries were sequenced on an Illumina HiSeq $^{\text {TM }} 2500$ according to the manufacturer's instructions.

Raw reads were processed to obtain clean data after removing reads that contained adapter or ploy- $\mathrm{N}$ as well as low quality reads. The Q20, Q30, and GC-content were calculated. Subsequently, the quality-filtered reads were used for downstream bioinformatics analysis. De novo assembly of the clean reads was conducted using Trinity software. Based on the short reads-assembling Trinity package with a k-mer length of 25 , the final clean reads were de novo assembled into unigenes. The obtained unigenes were checked against public databases for homology annotation, including the NCBI nonredundant protein (Nr) and nonredundant nucleotide (Nt) databases (http://www.ncbi.nlm.nih.gov/), Swiss-Prot (http://www.ebi.ac.uk/uniprot/), Gene Ontology (GO) (http://www.geneontology.org/), Clusters of Orthologous Groups (COG) (http://www.ncbi.nlm.nih.gov/COG/), and Kyoto Encyclopedia of Genes and Genomes (KEGG) (http://www.genome.jp/kegg/). All unigenes were subjected to blastx similarity search with a cutoff $E$-value of $1 \mathrm{e}^{-5}$ based on different databases.

Relative expression levels of the unigenes were assessed using the fragments per kilobase of exon per million fragments (FPKM). Statistical comparison between two different groups was conducted using DEGseq (version 1.18.0). A false discovery rate (FDR) of $<0.01$ and a FPKM ratio larger than 2 were set as the threshold for significant differential expression. GO was performed to identify the functional annotations of the DEGs. For GO enrichment analysis, we firstly mapped all DEGs to GO terms in the database (http://www.geneontology.org/) and then searched for GO terms that were 
significantly enriched using the GOseq R package. Moreover, KEGG was used for DEG pathway analysis.

To examine the reliability of the sequence of RNA molecules (RNA-Seq) results, ten candidate genes related to oxidative stress and immune response were selected for validation by qRT-PCR. Primers were designed using the Primer Premier 5 . The mud crab $18 \mathrm{~S}$ ribosomal RNA gene was selected as the internal control. Total RNA extraction from hepatopancreas in control and treatment groups was performed. The reverse transcription of the RNA was followed by PrimeScript RT reagent Kit With gDNA Eraser (Takara, Dalian, China) following the manufacturer's instructions. The quantitative reverse transcriptase (qRT-PCR) was amplified in a Bio-Rad RealTime PCR system (BioRad, US) using SYBR Green. The reaction mixtures were $20 \mu \mathrm{L}$, containing $2 \mu \mathrm{L}$ diluted cDNA sample $(50 \mathrm{ng} / \mu \mathrm{L}), 10 \mu \mathrm{L} 2 \times$ SYBR Premix Ex Taq, $0.4 \mu \mathrm{L}$ each of primer $(10 \mu \mathrm{M})$,

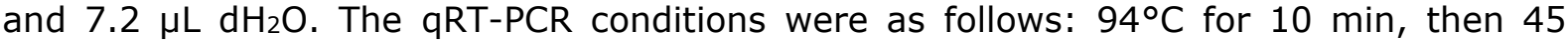
cycles at $95^{\circ} \mathrm{C}$ for $30 \mathrm{~s}, 60^{\circ} \mathrm{C}$ for $30 \mathrm{~s}$ and $72^{\circ} \mathrm{C}$ for $30 \mathrm{~s}$, followed by 10 min at $72^{\circ} \mathrm{C}$. Each sample was amplified in triplicates. At the end of each PCR reaction, the dissociation curve analysis of the amplification products was done. Relative gene expression levels were evaluated using $2^{-\Delta \Delta C T}$ method (Livak and Schmittgen, 2001).

Data were subjected to one-way ANOVA using SPSS 18.0 software (SPSS, Chicago, IL, USA), and the $\mathrm{p}$-values less than 0.05 were considered statistically significant.

\section{Results}

The effect of nitrite exposure on antioxidant activities and lipid peroxidation is shown in figure 1. The activity of SOD significantly increased at $6 \mathrm{~h}$ (Fig.1a). Compared to the control, the activity of SOD in the treatment group decreased significantly by $47 \%$ and $38 \%$ at $12 \mathrm{~h}$ and $24 \mathrm{~h}$ after nitrite exposure, respectively $(\mathrm{p}<0.05)$. Compared to the control group, the activity of CAT in the treatment group decreased significantly $76 \%$ and $65 \%$ at $12 \mathrm{~h}$ and $24 \mathrm{~h}$ after nitrite exposure, respectively $(\mathrm{p}<0.05)$ (Fig.1b). MDA content remained at control levels during the first $12 \mathrm{~h}(p>0.05)$. However, compared to the control group, MDA content in the treatment group increased significantly up to 1.47-fold at $24 \mathrm{~h}$ after $(\mathrm{p}<0.05)$ (Fig.1c).
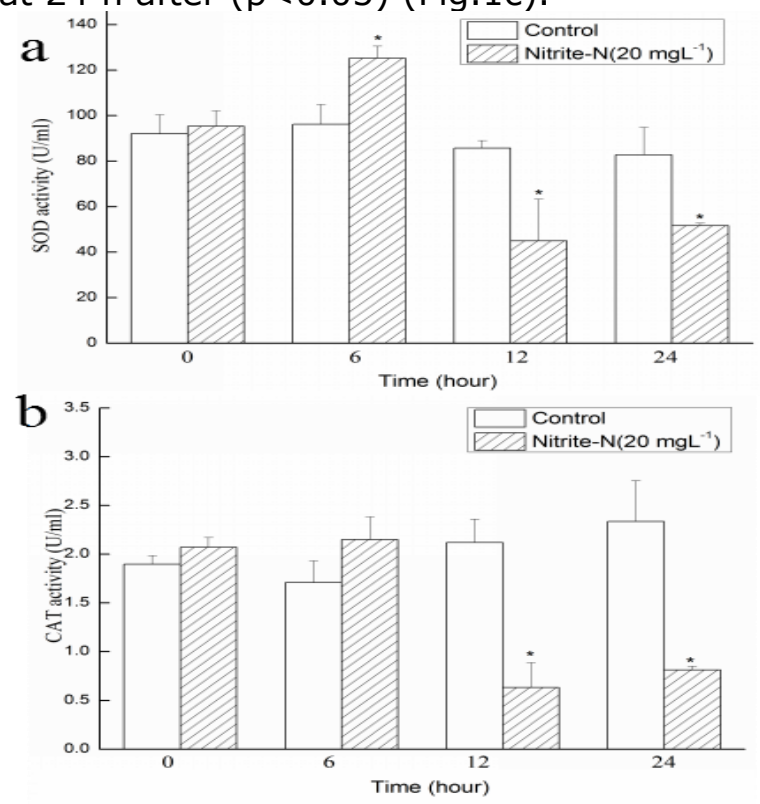

Fig.1 SOD activity (a), CAT activity (b) and MDA content (c) at $0,6,12$ and $24 \mathrm{~h}$ after nitrite exposure. Date was presented as the mean \pm SD. Asterisks indicated results that were significantly different from the control $(P<0.05)$.

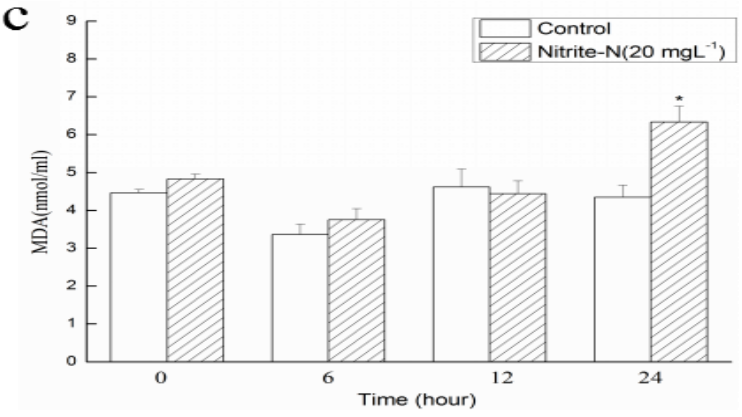


No abnormality was observed in histological sections from the control group (Fig. 2a). After nitrite exposure, in the hepatopancreas appeared vacuoles at $6 \mathrm{~h}$. The lumen was enlarged. Connective tissue disappeared at $12 \mathrm{~h}$ after nitrite exposure. Cell outlines were not clear at $12 \mathrm{~h}$ after nitrite exposure. (Fig.2).
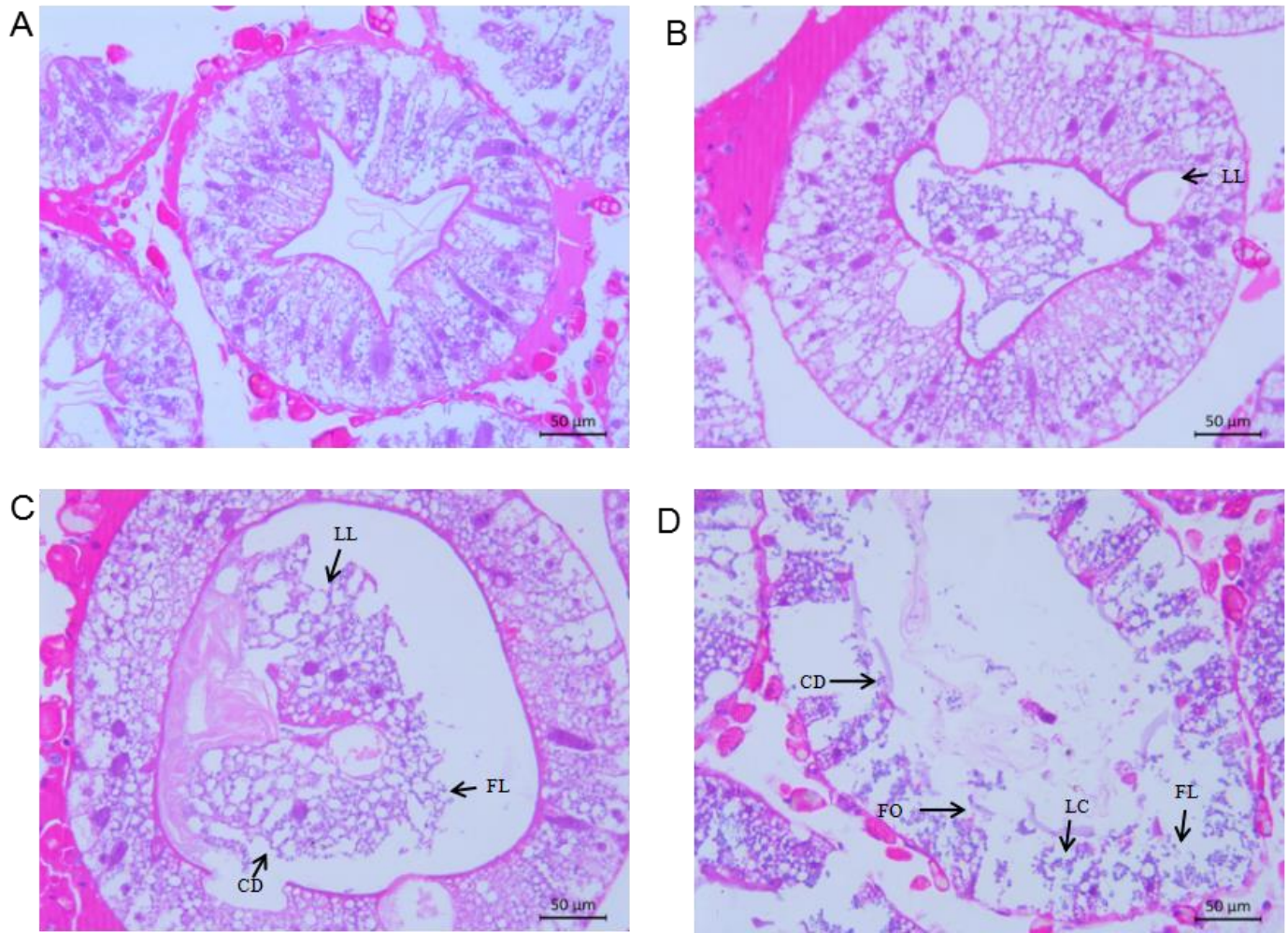

Fig. 2 Histological appearance of the hepatopancreas tissue after nitrite exposure. (A) $0 \mathrm{~h}$; (B) $6 \mathrm{~h}$; (C) 12 h; (D) 24 h. Fused lamellae (FL); Lumen largen (LL); Connective tissue disappeared (CD); Fuzzy cell outline (FO); lysis of cell (LC).

To identify the genes involved in the response of mud crab under nitrite stress, cDNA samples from the hepatopancreas in the control and nitrite exposed groups at $24 \mathrm{~h}$ were prepared and sequenced using an Illumina HiSeq 2500. The transcriptome assembly produced 105,136 unigenes with an average length of 847.6 bp a minimum length of $200 \mathrm{bp}$, a maximum length of $34,049 \mathrm{bp}$, and an N50 of $1648 \mathrm{bp}$ (Table 1). A length distribution analysis revealed that most of the unigenes were between 200 and $300 \mathrm{bp}$ in length.

Table 1. Summary of assembled transcripts and unigenes.

\begin{tabular}{|l|l|}
\hline Unigenes statistic & Date \\
\hline Total number of unigenes (Counts) & 105,136 \\
\hline Average length of unigenes (bp) & 847.60 \\
\hline Max length (bp) & 34,049 \\
\hline N50 length for unigenes (bp) & 1648 \\
\hline NR annotation (Counts) & 34,739 \\
\hline NT annotation (Counts) & 23,928 \\
\hline Swiss-Prot annotation (Counts) & 28,533 \\
\hline KEGG annotation (Counts) & 28,148 \\
\hline COG annotation (Counts) & 14,217 \\
\hline GO annotation (Counts) & 16,314 \\
\hline
\end{tabular}

Based on differential expression analysis, 11,638 unigenes were differentially expressed after nitrite exposure (FDR $<0.01$, fold change $>2$ ), including 7158 upregulated unigenes, and 4480 down-regulated unigenes. To further understand the transcriptional differences in response to nitrite exposure, all of the DEGs were then subject to GO classifications and KEGG pathway analysis. After annotation, a total of 1077 GO terms were assigned to 11638 DEGs. A total of 94 GO terms were considered 
significant over-representation with FDR $<0.05$. All DEGs were classified into three gene ontology categories, including molecular functions, cellular components, and biological processes (Fig.3).

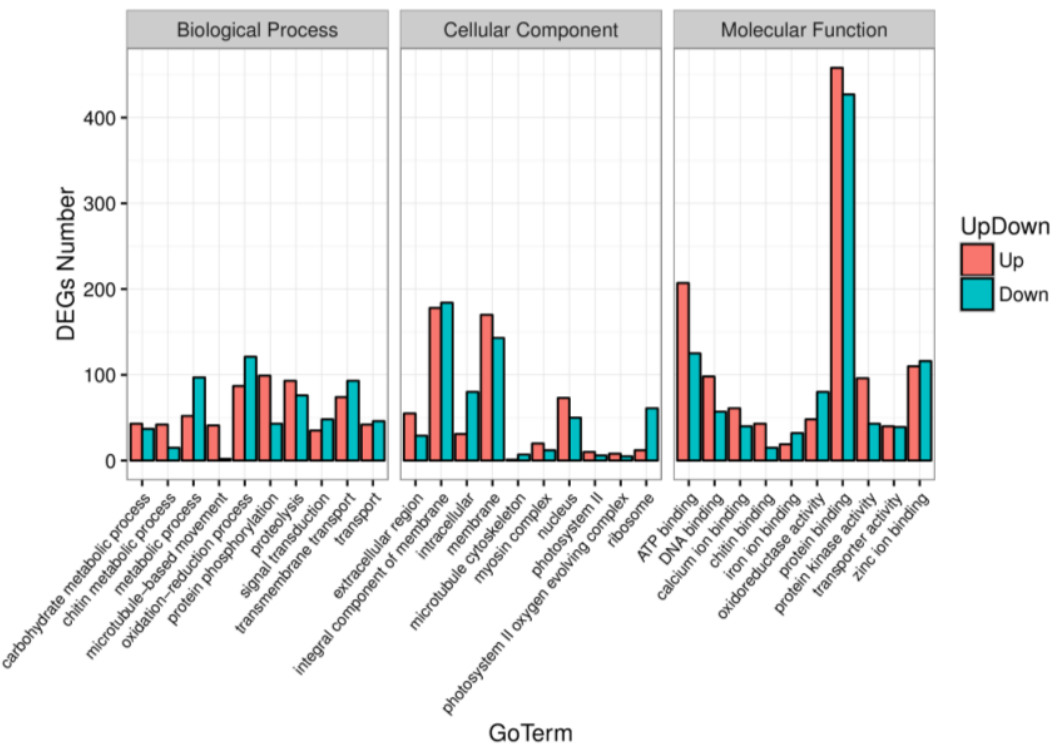

Fig.3

Gene classification based on Gene Ontology for DEGs.

In the biological process category, the top four subclasses were oxidation-reduction process, proteolysis, transmembrane transport, and metabolic process. In the cellular component category, the two most enriched subclasses were integral component of membrane, and membrane. While in the molecular function subclass, the most abundant category was protein binding. The levels of DEGs were also applied to KEGG pathway analysis. The DEGs were assigned to 299 KEGG pathways. The top 20 most enriched

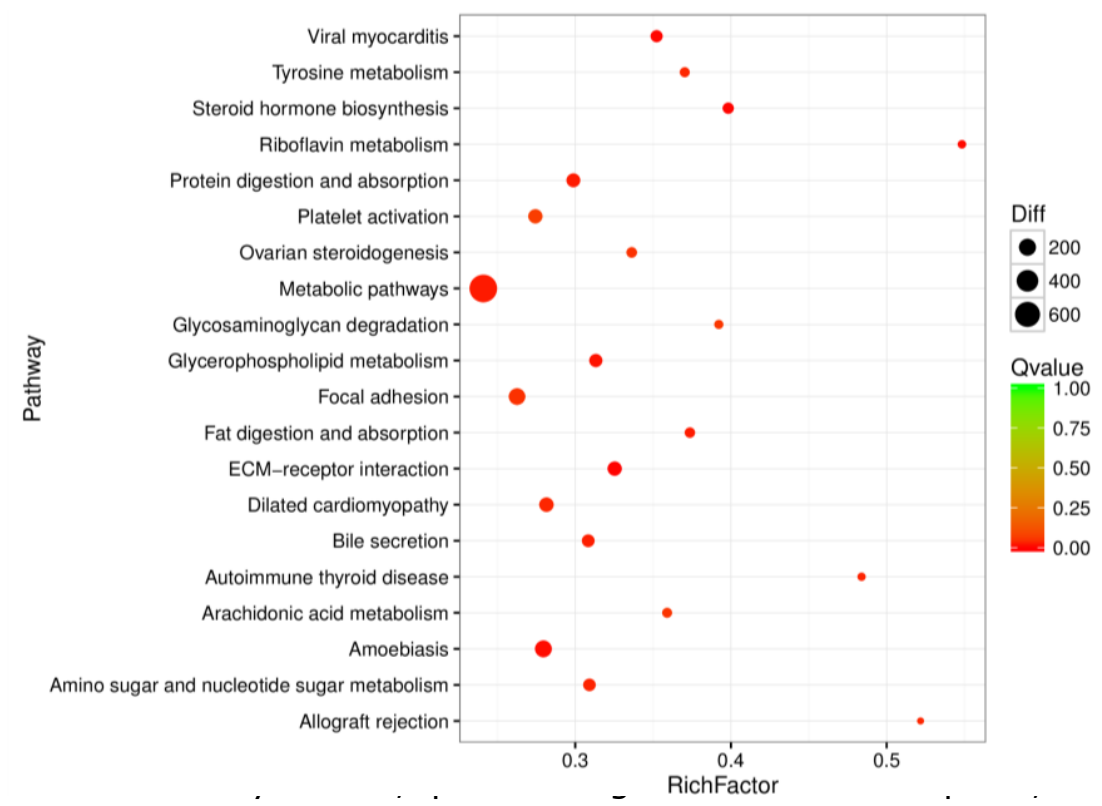
pathway terms are shown in Fig.4.

Fig.4 Scatter diagram of pathway enrichment for DEGs. In this scatter diagram, the top 20 pathways were listed, and rich factor is the ratio of DEGs in this pathway to all the genes in this pathway. response-related pathways, such as MAPK signaling pathway, Toll-like receptor signaling pathway, FoxO signaling pathway, NF-kB signaling pathway, p53 signaling pathway, NOD-like receptor signaling pathway, apoptosis, PPAR signaling, and Wnt signaling pathway, were also identified. These immune pathways may play important roles in the immunity of mud crab under nitrite stress responses. In this study, we focused on pathways related to oxidative stress and immune responses, such as FoxO signaling pathway, p53 signaling pathway and NF-kB signaling pathway (Table 2). Subsequently, 27 DEGs were screened, and distributed as shown in the heat map (Fig. 5). Our results show changes in immunity-related DGEs and pathways in response to nitrite stress as shown in Fig. 6. 
Table 2. List of DEGs involved in oxidative stress and immune responses between control and nitrite exposure group. " $\uparrow$ " represents the up-regulated genes. " $\downarrow$ " represents the down-regulated genes.

\begin{tabular}{|c|c|c|}
\hline $\begin{array}{l}\text { Pathway/Gene } \\
\text { FOXO signaling }\end{array}$ & Gene Title & log2FoldChange \\
\hline CAT & Catalase & $1.5 \uparrow$ \\
\hline Mn-SOD & Superoxide dismutase & $2.3 \uparrow$ \\
\hline BNIP3 & $\begin{array}{l}\text { BCL2/adenovirus E1B } 19 \mathrm{kDa} \text { protein interacting } \\
\text { protein } 3\end{array}$ & $2.9 \uparrow$ \\
\hline Atg12 & Autophagy-related protein 12 & $4.0 \uparrow$ \\
\hline INSR & Insulin receptor & $4.6 \uparrow$ \\
\hline PI3K & Phosphatidylinositide 3-kinases & $4.4 \uparrow$ \\
\hline SOS & RecA protein & $1.3 \uparrow$ \\
\hline FoxG1 & Forkhead box protein G1 & $-3.3 \downarrow$ \\
\hline JNK & c-Jun N-terminal kinases & $6.2 \uparrow$ \\
\hline P38 & p38 mitogen-activated protein kinases & $2.2 \uparrow$ \\
\hline IGF1R & The insulin-like growth factor 1 receptor & $-1.4 \downarrow$ \\
\hline \multicolumn{3}{|l|}{$\begin{array}{l}\text { P53 signaling } \\
\text { pathway }\end{array}$} \\
\hline P53 & Tumor protein p53 & $3.6 \uparrow$ \\
\hline P48 & DNA damage-binding protein & $1.6 \uparrow$ \\
\hline Sestrins & Sestrins & $1.9 \uparrow$ \\
\hline PIGs & p53 induced gene & $-3.4 \downarrow$ \\
\hline Cytc & Cytochromes C & $-4.9 \downarrow$ \\
\hline IGF-BP3 & Insulin-like growth factor 2 mRNA-binding protein 3 & $-4.3 \downarrow$ \\
\hline PTEN & Phosphatase and tensin homolog & $-2.0 \downarrow$ \\
\hline \multirow{3}{*}{\multicolumn{3}{|c|}{$\begin{array}{l}\text { NF-kB } \\
\text { signaling } \\
\text { pathway }\end{array}$}} \\
\hline & & \\
\hline & & \\
\hline Zap & Zeta-chain-associated protein kinase 70 & $7.6 \uparrow$ \\
\hline p50 & $\begin{array}{l}\text { Nuclear factor kappa-light-chain-enhancer of } \\
\text { activated B cells }\end{array}$ & $1.1 \uparrow$ \\
\hline MyD88 & Myeloid differentiation primary response 88 & $1.2 \uparrow$ \\
\hline PLC-y1 & Phospholipase C-y1 & $-1.5 \downarrow$ \\
\hline TRAF2 & TNF receptor-associated factor 2 & $-2.3 \downarrow$ \\
\hline TRAF6 & TNF receptor-associated factor 6 & $-5.7 \downarrow$ \\
\hline TAK1 & TGF $\beta$-activated kinase 1 & $-1.0 \downarrow$ \\
\hline CARMA1 & CARD-containing MAGUK protein 1 & $-1.8 \downarrow$ \\
\hline C-IAP1/2 & Cellular inhibitors of apoptosis & $5.1 \uparrow$ \\
\hline
\end{tabular}

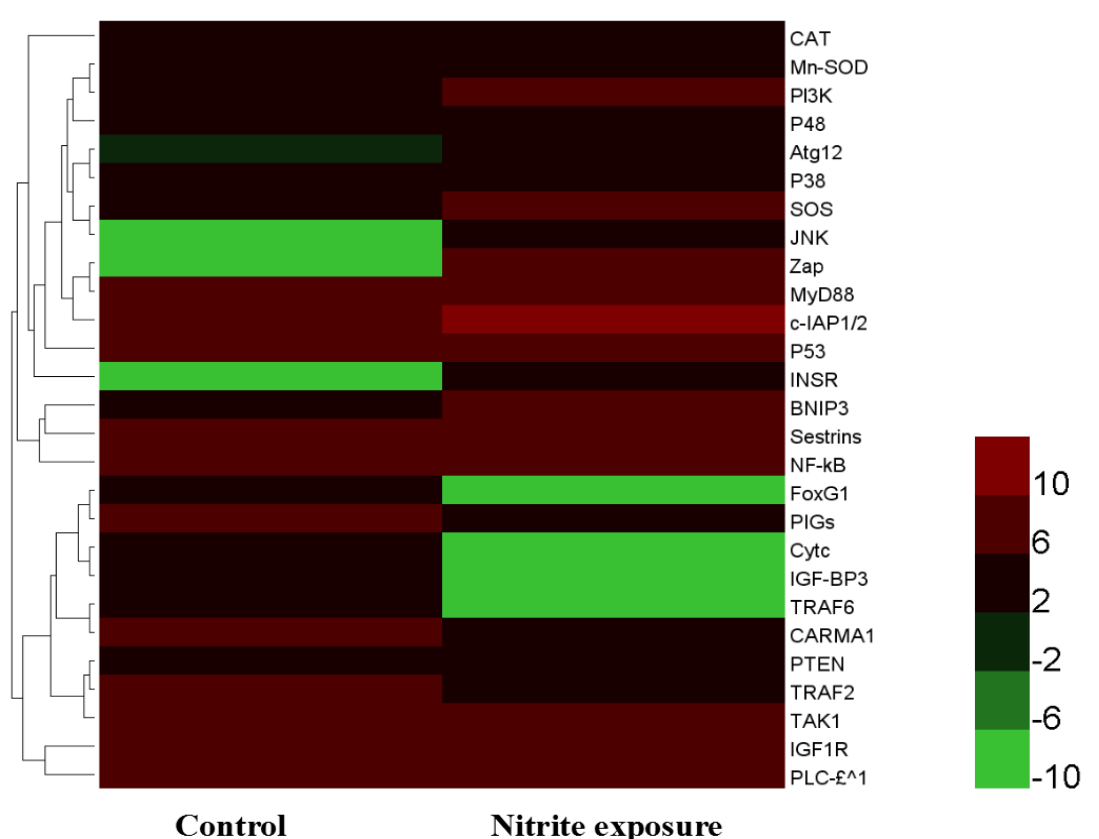

Control
Nitrite exposure
Fig.5 Heat map of DEGs related to immune responses (FOXO signaling pathway, p53 signaling pathway and NF-kB signaling pathway). The expression data was used in the form of $\log _{2}$ (FPKM) value. The red region indicates high expression levels in challenge to legend stimulation. The green region indicates low expression levels in challenge to legend stimulation. 


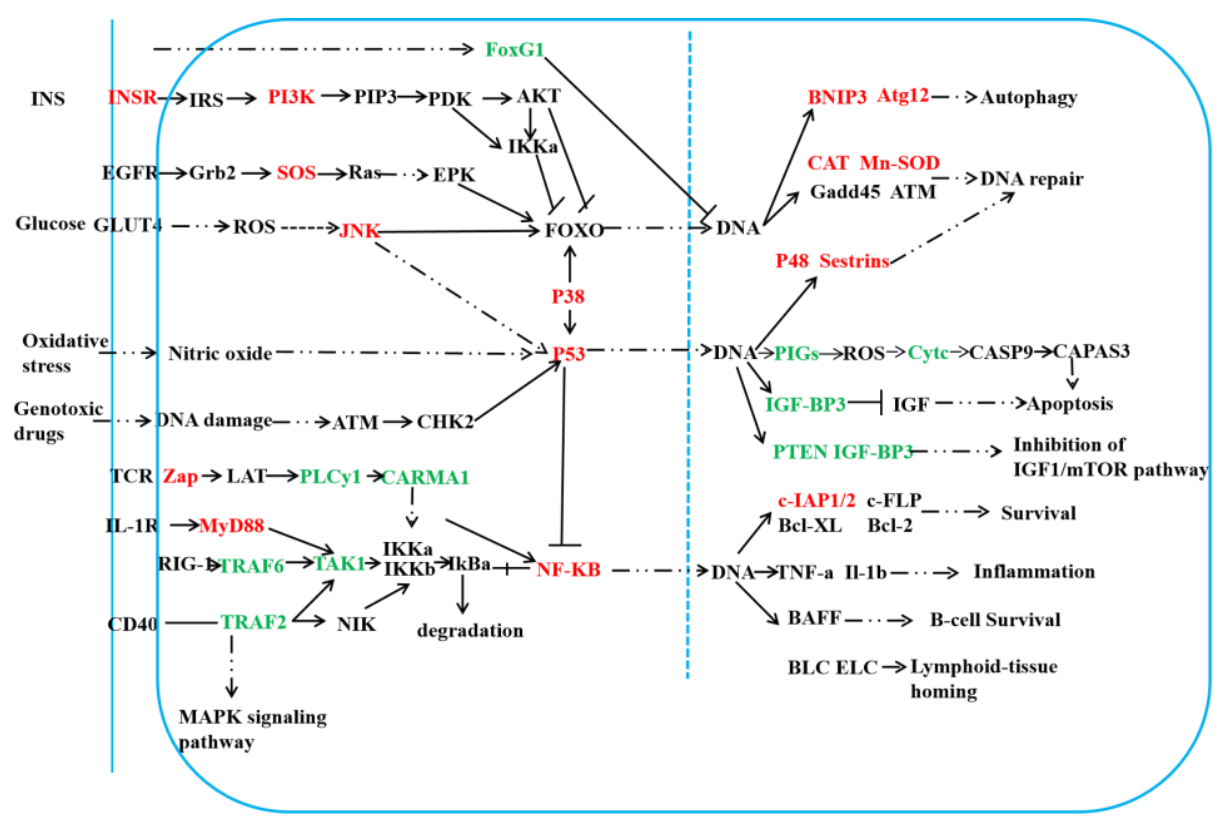

Fig.6 Changes of immunity-related DEGs and pathways after exposure to high environmental nitrite.

Red box: up-regulated unigene(s). Green box: down-regulated unigene(s).

The genes TRAF6, JNK, P38, and NF-kB play a vital role in innate immune response. CAT, Mn-SOD, and FoxG1 participate in antioxidant response. P53, cytc, and PIGs are involved in DNA repair and apoptosis. Thus, these key genes were selected for quantitative analysis by qRT-PCR to validate the accuracy of expression profile obtained from DGEs (Fig.7). Results showed that there were significance differences between the control and treatment groups $(p<0.05)$. Even though the slight differences in expression levels were not exactly the same, they showed the identical up-regulated and downregulated patterns of these ten genes in both assays. Results obtained from qRT-PCR were consistent with RNA-Seq analysis, confirming the reliability of the RNA-Seq expression analysis.

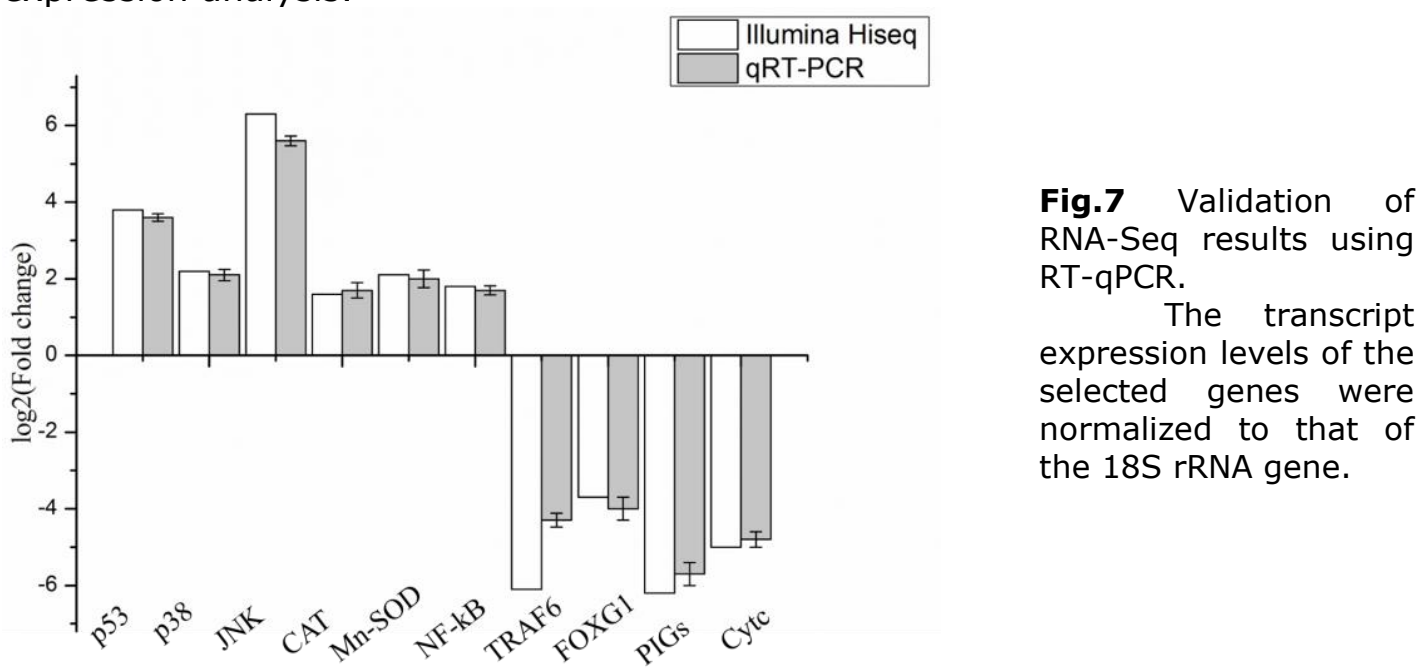

\section{Discussion}

It is well known that nitrite is generally toxic to aquatic organisms (Chen and Cheng, 1995; Tseng and Chen, 2004). The understanding of the molecular mechanism of nitrite toxicity in mud crabs is still quite limited. In the present study, we used the hepatopancreas of mud crab to perform a genome-wide investigation through transcriptional sequencing and gene expression profile analysis. It is the first transcriptomic analysis of mud crab with relevance to nitrite toxicity.

Previous studies showed that oxidative stress was one of the toxicity mechanisms of nitrite on aquatic organisms (Xian et al., 2011; Guo et al., 2013). Organisms developed detoxifcation and anti-oxidative defense to reduce oxidative stress. In this study, 27 DEGs were selected as directly involved in the immune-related pathways. These DEGs were divided into FoxO signaling pathway, p53 signaling pathway, and NF$\mathrm{kB}$ signaling pathway. In mammals, FoxO signaling has been linked to the regulation of immune system homeostasis (Bandyopadhyay and Medrano, 2003). 
It also controls numerous antioxidant gene expressions. According to our DEGs analysis, several anti-oxidative defense genes ( $\mathrm{Mn}$-SOD and CAT) were up-regulated at $24 \mathrm{~h}$ after nitrite exposure. Antioxidant enzymes (CAT and SOD) in Litopenaeus vannamei were upregulated to protect the hemocyte against nitrite exposure (Guo et al. 2013). SOD and CAT are the first line of defense against oxidative stress. SOD can convert the intracellular oxygen free radicals $\left(\mathrm{O}_{2}{ }^{-}\right)$into hydrogen peroxide $\left(\mathrm{H}_{2} \mathrm{O}_{2}\right)$ and molecular oxygen $\left(\mathrm{O}_{2}\right)$ (Rama and Manjabhat, 2014). CAT can eliminate $\mathrm{H}_{2} \mathrm{O}_{2}$ effectively thus reducing its toxic effect. The induced expression of CAT and SOD can protect cells against free radicals. However, our results indicated that there were no significant correlations between alterations of transcription and enzyme activities of SOD and CAT. This is similar to previous research on Wuchang bream under nitrite exposure (Sun et al., 2014). The mismatch between antioxidant enzymes activity and the transcriptional levels of genes is that antioxidant enzyme activity reflects total enzyme activity of the different isozymes, and the mRNA transcription level is limited to just one specified subtype of the antioxidant gene (Craig et al., 2007). Meanwhile, our results showed that SOD activity increased significantly at $6 \mathrm{~h}$, but then quickly decreased at $12 \mathrm{~h}$ and $24 \mathrm{~h}$ after nitrite exposure. At the same time, CAT activity was significantly inhibited after nitrite exposure. These results suggested that antioxidant enzymes protected the organism against oxidative damage induced by nitrite during the early exposure period. However, long-term exposure to nitrite stress may enhance the production of ROS, and then impair the antioxidants (Jia et al., 2015). Nitrite-induced oxidative stress can also attack polyunsaturated fatty acids, leading to lipid peroxidation (Romano and Zeng, 2013). MDA is a measure of terminal products of lipid peroxidation, which reflects the extent of oxidative damage in an organism (Jiang et al., 2017). In this study, MDA content increased significantly at $24 \mathrm{~h}$ under nitrite exposure, which was consistent with previous research (Jia et al., 2015). The increase in MDA levels may reflect the oxidative stress generated from nitrite exposure (Üner et al., 2011).

In our study, NF-kB were significantly up-regulated after nitrite exposure. The NF$\mathrm{kB}$ signaling pathway plays a vital role in inflammation, stress response, innate and adaptive immunity. NF-kB, an important transcription factor, is the center of the NF-kB signaling pathway. It can be activated by oxidative stress (Baeuerle, 1991). It is a key player in the inflammatory response by up-regulation of related genes involved in inflammation (Sen and Baltimore, 1986). Furthermore, NF-kB signaling is also a key player in anti-apoptotic signaling (Nam, 2006). Activation of NF-kB can lead to cell degeneration and death (Zhao et al., 2007). TRAF6 plays a vital role in the innate immune response. TRAF6 functions as a signal transducer in the NF-kB pathway. It also activates the JNK and p38 MAPK pathways and the IKK complex (Chung et al., 2002). In this study, we found significant down regulation of TRAF6 after nitrite exposure, suggested that nitrite exposure might impair immune-related gene expression.

The overproduction of ROS can modify DNA bases and cause strand scission by degrading the ribose ring. Previous studies indicated that environmental chemicals could lead to DNA damage (Mai et al., 2010; Cheng et al., 2015). The p53 signaling pathway plays an important role in cell cycles, genetic stability, and DNA repair (Levine, 1997). p53 is an important mediator of cell response to genotoxic stress. It can be activated by various stimuli such as hypoxia exposure, UV radiation, oxidative stress, and DNA damage (Vogelstein et al., 2000). Moreover, p53 has been proposed to function directly in P48 by interacting with damaged DNA (Fitch et al., 2003). Sestrins are a family of highly conserved stress-inducible proteins that participate in multiple diseases via regulating oxidative stress, cell apoptosis, and inflammatory response. Sestrins are p53target genes and confer resistance to p53-induced oxidative stress (Sun et al., 2014). Sestrins protect against oxidative stress-induced cell and tissue injury by reducing ROS levels and suppressing mTORC1 activity (Budanov and Karin, 2008). According to our DEGs analysis, p53 signaling pathway genes such as p53, P48, and sestrins were upregulated at $24 \mathrm{~h}$ after nitrite exposure. These results suggested that p53-sestrins signaling may play an important role in DNA repair. The p53 induced gene 3 (PIG3), another down-stream target of p53, is considered as a p53 dependent pro-apoptotic (Harris and Levine, 2005). PIG3 shares significant homology with oxidoreductases, 
playing an important role in the cell cycle, apoptosis, and genotoxic stress (Kotsinas et al., 2012). Cytochromes $\mathrm{c}$ (Cyt c) is a heme protein that is essential for aerobic respiration. The release of Cyt $c$ from mitochondria is a key initiative step in the activation of apoptosis initiation (Brown and Borutaite, 2008). In our study, we found that PIG3 and Cyt c were significantly down regulated after nitrite exposure. The down regulation of PIG3 and Cyt c expression may negatively affect apoptosis by decreasing the expression level of PIG3 and Cyt c gene.

In our study, nitrite exposure caused symptomatic damage such as fuzzy cell outline and lysis of cell. It is well known that oxidative stress is an important mediator of histopathological changes. In crustaceans, the hepatopancreas is central to metabolism and it is very susceptible to damage. Alterations in the hepatopancreas were useful markers to determine the toxicity of environmental stressors (Bernet et al., 1999). Histopathological damage such as intracellular edema, pycnotic nuclei, and fat droplets in the liver of Wuchang bream after acute exposure to nitrite were observed (Sun et al. 2014). High nitrite levels can even lead to tissue necrosis in aquatic animals (Dutra et al., 2017). Our results suggested that nitrite exposure could reduce the detoxification processes.

We investigated the molecular mechanism of nitrite-induced toxicity in mud crab. Our results indicated that nitrite stress caused oxidative stress and led to cellular and physiological impairment. The mud crab might counteract the toxicity of nitrite by altering the expression levels of genes of multiple immune-related pathways, including detoxification-related and anti-oxidative defense-related genes. Additionally, oxidative stress induced by nitrite might be impaired immune-related gene expression and disrupted metabolism. Improving the ability of immunity may enhance crustacean tolerance to nitrite exposure, via dietary supplemental antioxidant nutrients. Furthermore, this study discovered some genes which may provide candidate biomarkers for monitoring environmental pollutants.

\section{Acknowledgements}

This research was supported by the China Agriculture Research System (CARS-48), Marine Fisheries Technology and Extension System of Guangdong Province (A201701B01), Science and Technology Program Project of Guangzhou (201904010327).

\section{References}

Aebi, H., 1984. Catalase in vitro. Methods Enzymol. 105,121-126.

Baeuerle, P.A., 1991. The inducible transcription activator NF-kB: regulation by distinct protein subunits, Biochim. Biophys. Acta BBA-Rev.Cancer. 1072, 63-80.

Bandyopadhyay, D., Medrano, E.E., 2003. The emerging role of epigenetics in cellular and organismal aging. Exp. Gerontol. 38, 1299-1307.

Bernet, D., Schmidt, H., Meier, W., Brkhardt-Holm, P., Wahli, T., 1999. Histopathology in fish: proposal for a protocol to assess aquatic pollution. J. Fish Dis. 22, 25-34.

Brown, G.C., Borutaite, V., 2008. Regulation of apoptosis by the redox state of cytochrome c. Biochimica et Biophysica Acta. 1777, 877-881.

Budanov, A.V. Karin, M., 2008. p53 target genes sestrin1 and sestrin2 connect genotoxic stress and mTOR signaling. Cell 134,451-460.

Chand, R.K. Sahoo, P.K., 2006. Effect of nitrite on the immune response of freshwater prawn Macrobrachium malcolmsonii and its susceptibility to Aeromonas hydrophila, Aquaculture 258, 150-156.

Chen, J., Cheng, S., 1995. Changes of oxyhemocyanin and protein levels in the hemolymph of Penaeus japonicus exposed to ambient nitrite. Aquat. Toxicol. 33,215-226.

Chen, J.C., Chen, S.F., 1992. Effects of nitrite on growth and molting of Penaeus monodon juveniles. Comp. Biochem. Physiol. C 101, 453-458.

Chen, J.C., Cheng, S.Y., 1995a. Accumulation of urea in the haemolymph and ammonia excretion of Penaeus japonicus exposed to ambient nitrite. Comp. Biochem. Physiol.C 110, 1-6.

Cheng, C.H., Yang, F.F., Ling, R.Z., Liao, S.A., Miao, Y.T., Ye, C.X., Wang, A.L., 2015. Effects of ammonia exposure on apoptosis, oxidative stress and immune response in pufferfish (Takifugu obscurus). Aquat Toxicol. 164, 61-71

Chung, J.Y. Park, Y.C., Ye, H., Wu, H., 2002. All TRAFs are not created equal: common and distinct molecular mechanisms of TRAF-mediated signal transduction, J. Cell Sci. 115, 679-688. 
Craig, P.M., Wood, C.M., McClelland, G.B., 2007. Oxidative stress response and gene expression with acute copper exposure in zebrafish (Danio rerio). Am. J. Physiol. Regul. Integr. Comp. Physiol. 293, 1882-1892.

Deane, E.E., Woo, N.Y.S., 2007. Impact of nitrite exposure on endocrine, osmoregulatory and cytoprotective functions in the marine teleost Sparus sarba. Aquatic Toxicol, 82, 85-93.

Dutra, F.M., Rönnau, M., Sponchiado, D., Forneck, S.C., Freire, C.A., Ballester, E.L.C., 2017. Histological alterations in gills of Macrobrachium amazonicum juveniles exposed to ammonia and nitrite. Aquatic Toxicol,187,115-1.

Federation, W.E., Association, A.P.H., 2005. Standard Methods for the Examination of Water and Wastewater. American Public Health Association (APHA), Washington, DC, USA.

Fitch, M.E., Cross, I.V., Turner, S.J., Adimoolam, S., Lin, C.X., 2003. The DDB2 nucleotide excision repair gene product p48 enhances global genomic repair in p53 deficient human fibroblasts. DNA Repair. 2, 819-826.

Guo, H., Xian, J.A., Li, B., Ye, C.X., Wang, A.L., Miao, Y.T., Liao, S.A., 2013. Gene expression of apoptosis-related genes, stress protein and antioxidant enzymes in hemocytes of white shrimp Litopenaeus vannamei under nitrite stress. Comp Biochem Physiol C Toxicol Pharmacol. 157,366-71.

Guo, H., Ye, C.X., Wang, A.L., Xian, J.A., Liao, S.A., Miao, Y.T., Zhang, S.P., 2013. Trascriptome analysis of the Pacific white shrimp Litopenaeus vannamei exposed to nitrite by RNA-seq. Fish Shellfish Immunol. 35, 2008-2016.

Guo, Z.X., He, J.G., Xu, H.D., Weng, S.P., 2013. Pathogenicity and complete genome sequence analysis of the mud crab dicistrovirus-1. Virus Res, 171, 8-14.

Harris, S.L., Levine, A.J., 2005. The p53 pathway: positive and negative feedback loops, Oncogene 24, 2899-2908.

Hooper, A.B., Vannelli,T., Bergmann, D.J., Arciero, D.M.,1997. Enzymology of the oxidation of ammonia to nitrite by bacteria. Antonie van Leeuwenhoek, 71, 59-67.

Jensen, F.B., 2003. Nitrite disrupts multiple physiological functions in aquatic animals. Comp.Biochem. Physiol. A 135, 9-24.

Jia, R., Han, C., Lei, J.L., Liu, B.L., Huang, B., Huo, H.H., Yin, S.T., 2015. Effects of nitrite exposure on haematological parameters, oxidativestress and apoptosis in juvenile turbot (Scophthalmus maximus). Aquat Toxicol. 169, 1-9.

Jiang, W., Jiang, Z., M Du, M., Y Mao, Y., Li, J., 2017. Physiological and biochemical responses of Zhikong scallop, Chlamys farreri, to different thermal stressors. Aquacult Res, 48, 1-15.

Jiang, Q., Zhang, W., Tan, H., Pan, D., Yang, Y., Ren, Q., Yang, J., 2014. Analysis of gene expression changes, caused by exposure to nitrite, in metabolic and antioxidant enzymes in the red claw crayfish, Cherax quadricarinatus. Ecotoxicol Environ Saf. 423428.

Kotsinas, A., Aggarwal, V., Tan, E.J., Levy, B., Gorgoulis, V.G., 2012. PIG3: a novel link between oxidative stress and DNA damage response in cancer. Cancer Lett. 327, 97-102.

Lesser, M.P., 2006. Oxidative stress in marine environments: biochemistry and physiological ecology. Annu. Rev. Physiol. 68, 253-278.

Levine, A.J., p53, 1997. The cellular gatekeeper for growth and division, Cell 88 (3), 323-331.

Livak, K.J., Schmittgen, T.D., 2001. Analysis of relative gene expression data using real time quantitative PCR and the 2- ${ }^{\Delta C} \mathrm{CT}$ method. Methods 25, 402-408.

Mai, W.J., Yan, J.L., Wang, L., Zheng, Y., Xin, Y., Wang, W.N., 2010. Acute acidic exposure induces p53-mediated oxidative stress and DNA damage in tilapia (Oreochromis niloticus) blood cells. Aquat. Toxicol. 100, 271-281.

Mortazavi, A., Williams, B.A., Mccue, K. Schaeffer, L. Wold, B., 2008. Mapping and quantifying mammalian transcriptomes by RNA-Seq, Nat. Methods 5 (7), 621-628.

Nam, N.H., 2006. Naturally occurring NF-kappaB inhibitors. Mini Rev. Med. Chem. 6, 945-951. 
Ohkawa, H., Ohishi, N., Yagi, K., 1979. Assay for lipid peroxides in animal tissues by thiobarbituric acid reaction. Anal. Biochem. 95,351-358.

Palachek, R., Tomasso, J., 1984. Toxicity of nitrite to channel catfish (Ictalurus punctatus), tilapia (Tilapia aurea), and largemouth bass (Micropterus salmoides): evidence for a nitrite exclusion mechanism. Can. J. Fish. Aquat. Sci. 41, 1739-1744.

Peskin, A.V., Winterbourn, C.C., 2000. A microtiter plate assay for superoxide dismutase using a water-soluble tetrazolium salt (WST-1). Clin. Chim. Acta.293, 157166.

Rama, S., Manjabhat, S.N., 2014. Protective effect of shrimp carotenoids against ammonia stress in common carp Cyprinus carpio. Ecotoxicol. Environ. Saf. 107, 207-213.

Romano, N., Zeng, C., 2013. Toxic effects of ammonia, nitrite, and nitrate to decapod crustaceans: a review on factors influencing their toxicity, physiological consequences, and coping mechanisms, Rev. Fish. Sci. 21 (1), 1-21.

Sen, R., Baltimore, D., 1986. Multiple nuclear factors interact with the immunoglobulin enhancer sequences, Cell. 46, 705-716.

Stadtman, E., Levine, R., 2003. Free radical-mediated oxidation of free amino acids and amino acid residues in proteins. Amino Acids. 25, 207-218.

Sun, G., Xue, R., Yao, .F, Liu, D., Huang, H., Chen, C., Li, Y., Zeng, J., Zhang, G., Dong, Y., Liu, C., 2014. The critical role of Sestrin 1 in regulating the proliferation of cardiac fibroblasts. Arch Biochem Biophys.542, 1-6.

Sun, S., Ge, X., Xuan, F., Zhu, J., Yu, N., 2014. Nitrite-induced hepatotoxicity in Bluntsnout bream (Megalobrama amblycephala): The mechanistic insight from transcriptome to physiology analysis. Environ Toxicol Pharmacol. 37 (1), 55-65.

Sun, S., Ge, X., Zhu, J., Xuan, F., Jiang, X., 2014. Identification and mRNA expression of antioxidan enzyme genes associated with the oxidative stress response in the Wuchang bream (Megalobrama amblycephala Yih) in response to acute nitrite exposure. Comp Biochem Physiol C Toxicol Pharmacol.159, 69-77.

Tseng, I.T., Chen, J.C., 2004. The immune response of white shrimp Litopenaeus vannamei and its susceptibility to Vibrio alginolyticus under nitrite stress. Fish Shellfish Immunol. 17, 325-333

Üner, N., Oruc, E.ö., Canli, M., Sevgler, Y., 2001. Effects of cypermethrin on antioxidant enzyme activities and lipid peroxidation in liver and kidney of the freshwater fish, Oreochromis niloticus and Cyprinus carpio (L.). Bull. Environ. Contam. Toxicol. 67, 657-664.

Vogelstein, B., Lane, D., Levine, A.J., 2000. Surfing the p53 network, Nature 408, 307-310.

Wang, L., Liu, P., Wan, Z.Y., Huang, S.Q., Wen, Y.F., Lin, G., Yue, G.H., 2016. RNA-Seq revealed the impairment of immune defence of tilapia against the infection of Streptococcus agalactiae with simulated climate warming. Fish Shellfish Immunol. 55, 679-689.

Wang, W.N., Wang, A.L., Zhang, Y.J., Li, Z.H., Wang, J.X., Sun, R.Y., 2004. Effects of nitrite on lethal and immune response of Macrobrachium nipponense. Aquaculture 232, 679-686.

Weng, S.P., Guo, Z.X., Sun, J.J., Chan, S.M., He, J.G., 2007. A reovirus disease in cultured mud crab, Scylla serrata, in southern China. J Fish Dis. 30, 133-139.

Woo, N.Y.S., Chiu, S.F., 1997. Metabolic and osmoregulatory responses of the sea bass Lates calcarifer to nitrite exposure. Environ. Toxicol. Water Qual. 12, 257-264.

Xian, J.A.,Wang, A.L., Chen, X.D., Gou, N.N., Miao, Y.T., Liao, S.A., Ye, C.X., 2011. Cytotoxicity of nitrite on haemocytes of the tiger shrimp, Penaeus monodon, using flow cytometric analysis. Aquaculture 317, 240-244.

Yu, J., Ji, X., Wang, X., Li, T., Wang, H., Zeng, Q., 2019. Identification and characterization of differentially expressed genes in hepatopancreas of oriental river prawn Macrobrachium nipponense under nitrite stress. Fish Shellfish Immunol. 144-154.

Zhao, T., Yang, L., Sun, Q., Arguello, M., Ballard, D.W., Hiscott, J., Lin, R., 2007. The NEMO adaptor bridges the nuclear factor-kappaB and interferon regulatory factor signaling pathways. Nat. Immunol. 8, 592-600. 